\title{
First trimester serum biomarkers to predict gestational diabetes in a high-risk cohort: Striving for clinically useful thresholds
}

Siobhan M. Corcoran

Royal College of Surgeons in Ireland, siobhancorcoran@rcsi.ie

Natalie Achamallah

Royal College of Surgeons in Ireland

John O'Loughlan

Rotunda Hospital, Ireland

See next page for additional authors

Follow this and additional works at: https://arrow.tudublin.ie/scschbioart

Part of the Obstetrics and Gynecology Commons

\section{Recommended Citation}

Corcoran SM, Achamallah N, Loughlin JO, Stafford P, Dicker P, Malone FD, Breathnach F. First trimester serum biomarkers to predict gestational diabetes in a high-risk cohort: Striving for clinically useful thresholds. Eur J Obstet Gynecol Reprod Biol. 2018 Mar;222:7-12. doi: 10.1016/j.ejogrb.2017.12.051. Epub 2018 Jan 1. PMID: 29328940.

This Article is brought to you for free and open access by the School of Biological Sciences at ARROW@TU Dublin. It has been accepted for inclusion in Articles by an authorized administrator of ARROW@TU Dublin. For more information, please contact arrow.admin@tudublin.ie, aisling.coyne@tudublin.ie,gerard.connolly@tudublin.ie. Funder: Friends of the Rotunda

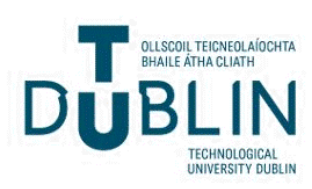




\section{Authors}

Siobhan M. Corcoran, Natalie Achamallah, John O'Loughlan, Philip Stafford, Pat Dicker, Fergal D. Malone, and Fionnuala Breathnach

This article is available at ARROW@TU Dublin: https://arrow.tudublin.ie/scschbioart/306 
Full length article

\title{
First trimester serum biomarkers to predict gestational diabetes in a high-risk cohort: Striving for clinically useful thresholds
}

\author{
Siobhan M. Corcoran ${ }^{\mathrm{a}, *}$, Natalie Achamallah ${ }^{\mathrm{a}}$, John O' Loughlin ${ }^{\mathrm{b}}$, Philip Stafford ${ }^{\mathrm{c}}$, \\ Pat Dicker ${ }^{\mathrm{d}}$, Fergal D. Malone ${ }^{\mathrm{a}}$, Fionnuala Breathnach ${ }^{\mathrm{a}}$ \\ ${ }^{a}$ RCSI Department of Obstetrics and Gynaecology, Ireland \\ ${ }^{\mathrm{b}}$ Department of Laboratory Medicine, Rotunda Hospital, Ireland \\ c Department of Biochemistry, Dublin Institute of Technology, Ireland \\ ${ }^{\mathrm{d}}$ RCSI Department of Public Health and Epidemiology, Ireland
}

\section{A R T I C L E I N F O}

\section{Article history:}

Received 25 August 2017

Received in revised form 18 December 2017

Accepted 30 December 2017

\section{Keywords:}

Gestational Diabetes

Screening

Biomarkers

First trimester

\begin{abstract}
A B S T R A C T
Objectives: Screening and diagnosis of gestational diabetes (GDM) has been a source of controversy. The prevalence has increased in line with an obesity epidemic and a trend towards delayed child-bearing. Treatment of even modest glycaemic impairment in pregnancy has been shown to be beneficial in preventing its clinical sequalae. However the cumbersome nature and timing of the oral glucose tolerance test coupled with debate around universal versus risk factor based screening have been problematic. This group aimed to investigate a panel of biomarkers which have shown promise in the literature to predict GDM from the first trimester in a group of high risk women.

Methods: Serum samples were drawn on 248 women deemed at risk of GDM before 15 weeks' gestation to measure C-reactive protein, sex hormone binding globulin, adiponectin and 1,5 anhydroglucitol. Patients underwent an oral glucose tolerance test as per IADPSG criteria at 28 weeks' gestation. Multiple logistic regression was used to examine the link between incidence of GDM and early pregnancy serum biomarkers.

Results: Adiponectin levels in the first trimester are independently linked to the risk of GDM. Serum adiponectin $<8.9 \mu \mathrm{g} / \mathrm{ml}$ gives an odds ratio of 3.3 for GDM.Mean 1,5 AG levels are significantly lower in those that go on to develop GDM. SHBG levels measured in the first trimester were linked to the risk of GDM. However, this was no longer statistically significant once BMI, ethnicity and family history were taken into consideration. First trimester measurement of CRP is not a useful indicator of GDM risk. Conclusions: First trimester measurement of Adiponectin and 1,5 Anhydroglucitol are potential early biomarkers for the later onset of GDM. Risk stratification using these biomarkers may facilitate early diagnosis and management of GDM to mitigate against its complications.
\end{abstract}

(c) 2018 Elsevier B.V. All rights reserved.

\section{Introduction}

Screening and diagnosis of GDM have presented a challenge for the last four decades or more. Research in this area is marred by controversy as different studies have used varying diagnostic criteria, rendering comparison of methodology and results problematic. GDM has come sharply in to the clinician's focus in recent years, owing to the obesity epidemic, delayed childbearing and an increasingly ethnically diverse pregnant population [1-4]. The clinical sequelae [5-7], high prevalence of glucose intolerance

\footnotetext{
* Corresponding author at: RCSI Department of Obstetrics and Gynaecology, Rotunda Hospital, Parnell Square Dublin 1, D01 K4H0, Ireland.

E-mail address: siobhancorcoran@rcsi.ie (S.M. Corcoran).
}

in the population and evidence of improved outcomes with even modest prenatal intervention, may make the case for universal screening clear $[5,8,9]$. However, universal screening may equally result in an increased healthcare burden and over-medicalisation of pregnancy.

In most cases of GDM, lifestyle interventions are effective at achieving normo-glycaemia throughout pregnancy [10,12]. A small subgroup will require hypoglycaemic agents to achieve this goal and reduce the risk of adverse outcome. Early intervention is key to optimising pregnancy outcome for mother and baby. Traditionally, screening for GDM involves selecting a sub-group of the population deemed to be at high risk of developing the condition. Risk factors include a family history of diabetes, those with a BMI $>30$, a history of polycystic ovarian syndrome or non-Caucasian ethnicity. Women with history-based or demographic risk factors 
undergo a formal oral glucose tolerance test (OGTT) at 28 weeks' gestation. The National Institute for Clinical Excellence (NICE) in the UK quote a $60 \%$ detection rate for a $40 \%$ false positive rate using this risk-factor based screening approach.

Early identification of women that will likely go on to develop GDM would allow the introduction of targeted dietary and lifestyle interventions in a more timely and effective manner, leading to potentially improved outcomes. There have been many biomarkers which have shown promise in the field of GDM [13-16]. Aiming to build on evidence already available in this field, our team elected to investigate a panel of promising biomarkers measured in the first trimester in a group of women deemed to be at high risk of GDM. We aimed to investigate the link between 1 st trimester serum C-Reactive protein (CRP), Sex hormone binding globulin (SHBG), adiponectin and 1,5 Anhydroglucitol(1,5AG) and subsequent onset of GDM in a high risk cohort.

\section{Methods}

This study was conducted at a single centre in the Rotunda Hospital Dublin between January 2014 and October 2015. The Rotunda is a large tertiary and stand-alone maternity unit delivering more than 8500 births annually. Institutional Review Board approval was sought and granted. Patients were considered eligible if they were less than 15 weeks' gestation at enrolment and had one or more of the following risk factors for gestational diabetes identified at the registration visit;

- $\mathrm{BMI} \geq 30 \mathrm{~kg} / \mathrm{m} 2$ (as objectively measured by midwife; not selfreported)

- Maternal age $>40$ years

- Ethnicity - Indian, Pakistani, South East Asian, Middle eastern, Afro-Caribbean

- History of Polycystic Ovarian Syndrome (PCOS)

- Family history of first degree relative with type 2 diabetes

- Previous macrosomic baby (>4 kg birthweight)

- Previous unexplained stillbirth

\section{Exclusion criteria}

- Persistent fasting glycosuria (as this merits first trimester screening for GDM and implies a risk of pre-existing type II diabetes)
- Gestational diabetes in a prior pregnancy (as the recurrence risk for gestational diabetes approximately 65\%)

- Twin Pregnancy. (due to potential difficulty in interpreting serum biomarkers)

Study participants underwent non-fasting serum testing of CRP, SHBG, Adiponectin and 1,5 AG taken alongside first pre-natal visit blood tests. All patients were scheduled for a $75 \mathrm{~g}$ OGTT at 28 weeks gestation. Using International Association of Diabetes and Pregnancy Study Group (IADPSG) criteria, the patient was considered to have GDM if the fasting serum glucose was greater than or equal to $5.1 \mathrm{mmol} / \mathrm{L}$, the 1 -h serum glucose was greater than or equal to $10.0 \mathrm{mmol} / \mathrm{L}$ or if the 2 -h post prandial serum glucose was greater than or equal to $8.5 \mathrm{mmol} / \mathrm{L}$. One or more of the readings above-threshold was sufficient for a diagnosis of GDM. Body mass index (BMI) was objectively measured. Clinicians and patients were blinded to biomarker results. Data was analysed using a univariate and multivariate logistic regression to determine the odds ratio of a positive OGTT for a given biomarker result. ROC curves were generated for those biomarkers with significant results in an attempt to define clinically useful thresholds. SPSS was used to perform statistical analyses and $\mathrm{p}<0.05$ was considered significant.

With regard to a power calculation we estimated that approximately $20 \%$ of our high risk cohort would screen positive for GDM. This study was largely exploratory (most especially with regard to 1,5 AG) which makes a detailed and exact power calculation difficult. We had no pilot study upon which to base our calculations and because the literature is variant and contradictory in some aspects regarding each biomarker, supposing the effect size was challenging. Using a background population incidence of 12\% GDM (based on ATLANTIC DIP results) and taking the probability of a type 1 error $(\alpha)$ to be 0.05 , while taking the power at $90 \%$ we determined we would need a sample size of 206 to show a statistically significant difference between screen positive and screen negative groups. We chose to extend our recruitment significantly beyond this due to the factors discussed above (Figs. 1-3 Tables 1 and 2).

\section{Discussion}

Gestational Diabetes (GDM) is an increasingly common complication of pregnancy [2]. It confers a risk of adverse outcome upon both the mother (increased rate of obstetric intervention, operative

Results

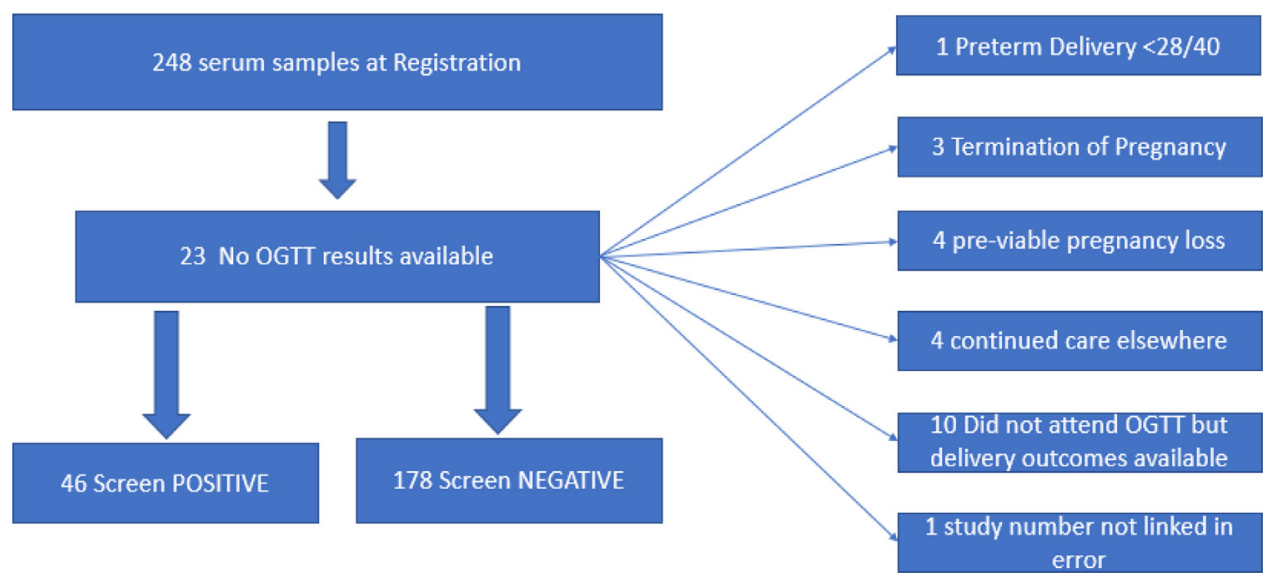

Fig 1. Study Population. 


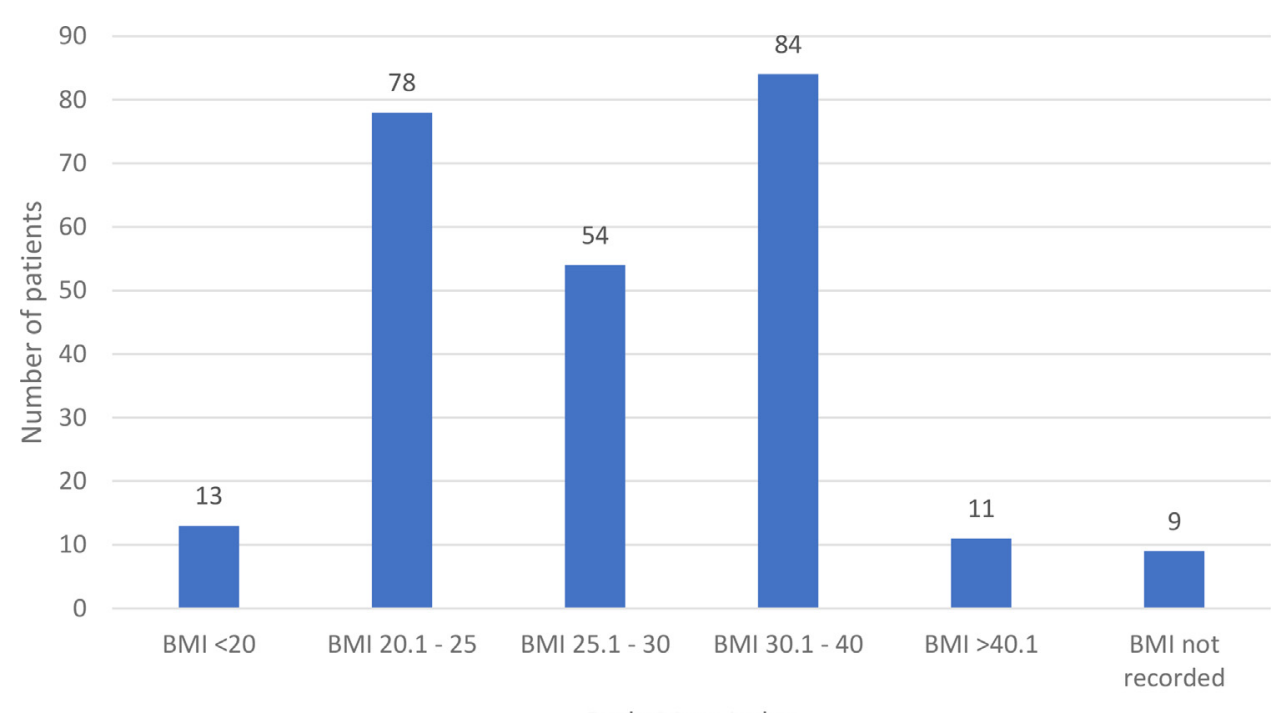

Body Mass Index

Fig 2. Body Mass Indices of the cohort.

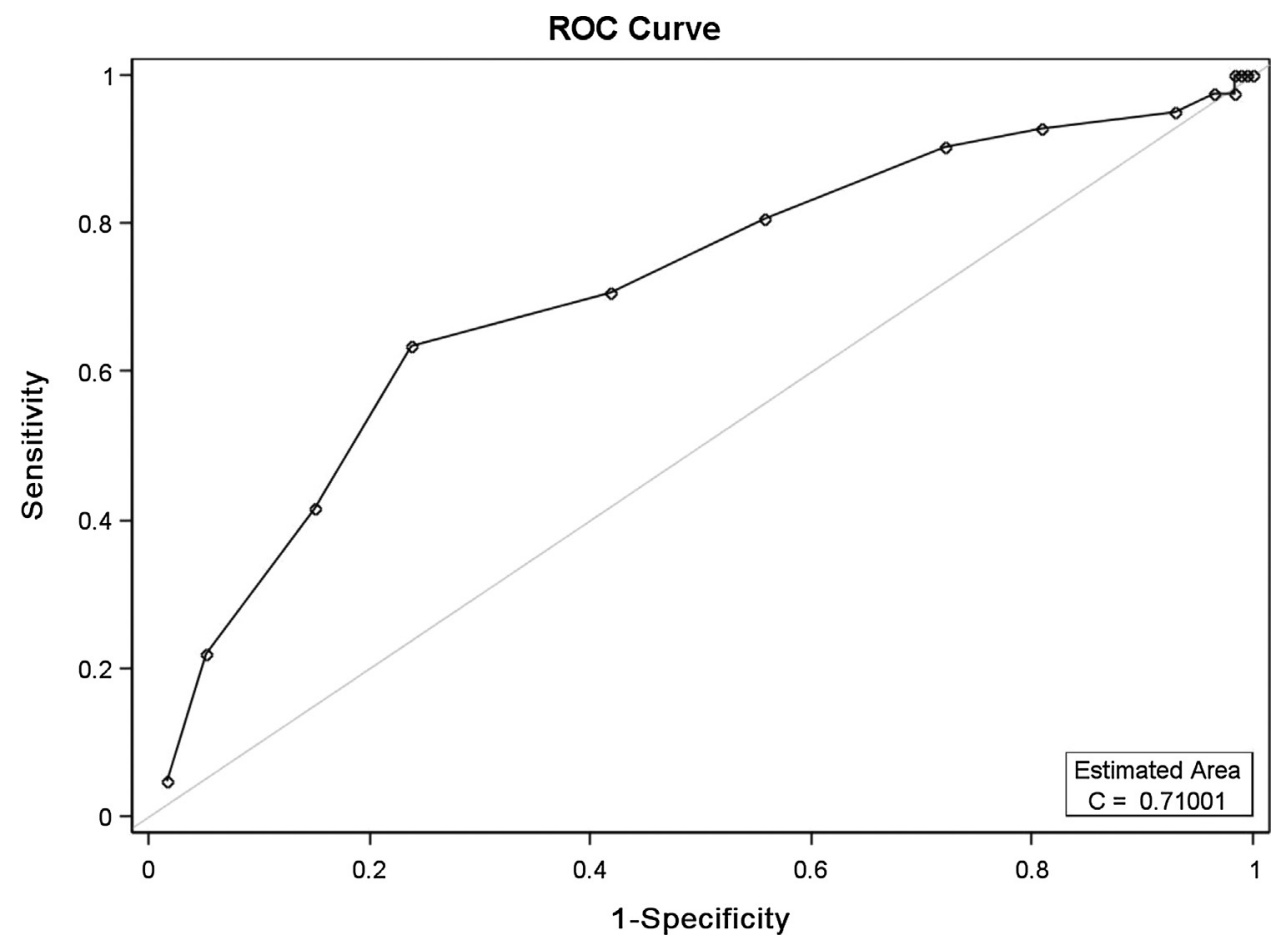

Fig 3. ROC curve of Adiponectin values in the first trimester as a predictor of GDM status.

Table 1

Risk factors for GDM in screen positive and screen negative groups.

\begin{tabular}{|c|c|c|c|}
\hline & GDM Screen Positive $N=46$ & GDM Screen Negative N = 178 & p-value \\
\hline Average Age (years) & 33.4 & 32.7 & $\mathrm{p}=0.65$ \\
\hline Average BMI $\left(\mathrm{kg} / \mathrm{m}^{2}\right)$ & 31.7 & 27.8 & $p=0.043$ \\
\hline Average number of Risk Factors for GDM & 1.65 & 1.25 & $\mathrm{p}=0.61$ \\
\hline
\end{tabular}

delivery and anal sphincter injury) and the fetus (macrosomia, shoulder dystocia, birth injuries and long term risks of childhood obesity and diabetes) [5-7]. There is a trend towards identifying pregnancies at risk of complications earlier in many fields of obstetric practice [13]. Examples include first trimester screening for aneuploidy, risk-factor and sonographic screening for intrauterine growth restriction and many biomarkers have been investigated in the prediction of pre-eclampsia. This approach allows us to identify and focus on a subgroup of high risk pregnancies whilst allowing the majority to proceed with minimal intervention and cost. 
Table 2

Basic statistical parameters of the biomarkers.

\begin{tabular}{|c|c|c|c|c|}
\hline & SHBG (nmol/L) & $\mathrm{CRP}(\mathrm{mg} / \mathrm{L})$ & Adiponectin $(\mu \mathrm{g} / \mathrm{mL})$ & 1,5 AG $(\mu \mathrm{g} / \mathrm{mL})$ \\
\hline Number of samples analysed & 219 & 222 & 213 & 213 \\
\hline Mean Value (st dev) & $281(88.8)$ & $5.6(5.8)$ & $8.9(2.9)$ & $15.9(6.3)$ \\
\hline Median Value & 281 & 3.9 & 9 & 15.9 \\
\hline Mode Value & 282 & 1 & 8 & 10.9 \\
\hline Range & $67-644$ & $1-62$ & $4-31$ & $2-35.3$ \\
\hline
\end{tabular}

\section{Table 3}

Logistic Regression Analysis of first trimester biomarker values and likelihood of a screen positive OGTT. Odds ratios correspond to 1 standard deviation increase in a biomarker level. Adjusted analysis* is multiple logistic regression adjusting for BMI, ethnicity and family history of diabetes.

\begin{tabular}{|c|c|c|c|c|}
\hline \multirow[t]{2}{*}{ Biomarker } & \multicolumn{2}{|c|}{ Unadjusted Analysis } & \multicolumn{2}{|l|}{ Adjusted Analysis* } \\
\hline & OR $(95 \% \mathrm{CI})$ & P-value & OR $(95 \% \mathrm{CI})$ & P-value \\
\hline SHBG & $0.64(0.44-0.94)$ & 0.021 & $0.78(0.52-1.17)$ & 0.231 \\
\hline CRP & $1.34(0.98-1.82)$ & 0.063 & $1.01(0.69-1.47)$ & 0.952 \\
\hline Adiponectin & $0.47(0.31-0.69)$ & $<0.001$ & $0.64(0.41-0.99)$ & 0.047 \\
\hline $1,5 \mathrm{AG}$ & $0.71(0.50-1.02)$ & 0.063 & $0.57(0.37-0.90)$ & 0.016 \\
\hline
\end{tabular}

In this study, we identified four biomarkers which have demonstrated early promise in the literature as biomarkers for GDM [17]. We investigated their utility in predicting GDM in a high risk first trimester cohort.

CRP is a protein found in plasma which rises in response to inflammation and as part of the acute phase response. It is synthesised in the liver and has a half-life of $48 \mathrm{~h}$ which is constant. CRP levels vary depending on the rate of production and so correlate to the severity of the precipitating cause. CRP has also been independently investigated as a biomarker capable of predicting the onset of gestational diabetes. Wolf et al. [18] found that in a prospective nested case-control study where 43 women subsequently developed GDM; higher levels of CRP in the first trimester positively correlated with the onset of disease and the difference between the two groups was statistically significant. However, once the results were adjusted for obesity the association was attenuated leading the authors to recommend that further larger studies should evaluate this in more detail. CRP is a biomarker that can change in great magnitude from day to day and a single measurement at a point in time may not truly reflect the background inflammatory metabolic milieu of the subject. Our CRP data was not normally distributed, with a small number of very high results skewing the data. This may have affected our results. Based on our study and the conflicting findings of the current body of available research on CRP use as a biomarker for GDM, we feel it is unlikely to prove a useful tool to this end. A serious draw back to employing CRP as a screening tool is its non-specific nature and the plethora of unnecessary investigations which may ensue following a high result. Routinely measuring CRP in otherwise healthy asymptomatic women would likely lead to unnecessary diagnostics, unfounded patient anxiety and increased healthcare costs.

1,5 Anhydroglucitol is a monosaccharide which is present in almost all foods. 1,5 AG is in a steady state normally and almost completely reabsorbed within the renal tubules. Serum levels decrease during periods of hyperglycaemia due to increased urinary excretion. In the absence of hyperglycaemia the levels return to normal within 14 days. It may be used therefore to detect short term excursions in glycaemia which may not be represented by the traditional methods such as measurement of HbA1c and fructosamine. It has been shown to more accurately detect hyperglycaemic episodes than other markers when compared to continuous glucose monitoring packs (CGM) [19]. Initially this marker was not investigated in pregnancy as it was felt that physiological changes in kidney function and haemodynamic parameters may invalidate the results but Dworacka et al. recently demonstrated it to be a reliable indicator of short term hyperglycaemic episodes in pregnancy in a group of 55 women (both with gestational and pregestational diabetes) and suggests its use as an adjunct to HbA1c [20]. Recently a study of 85 patients with a mixture of T1DM, T2DM and gestational diabetes were investigated by Delaney et al. They found an inverse relationship between the 1,5 AG levels measured at 28 weeks gestation and the birthweight of the infants. The group conclude that this marker "may be useful in the assessment of glycaemic control in pregnancy in addition to A1c" [21]. Nowak et al. also published on the inverse relationship between mid-trimester 1,5 AG levels and birthweight in women with T1DM. They found 1,5 AG to be a stronger predictor of macrosomia than HbA1C [22]. There is only one study we identified that attempts to determine the normal 1,5 AG values in diabetic, non-diabetic pregnancies and non-pregnant women. Tetsuo et al. showed the level of 1,5 AG in the nonpregnant woman was $18.6 \pm 5.2 \mathrm{mg} / \mathrm{L}($ mean $\pm \mathrm{SD})$. While the group noted that levels began to decrease in parallel with gestation from about 9 weeks they do not present reference ranges for pregnancy [23].

Table 3 shows that each one standard deviation increase in 1,5 AG measured in the 1 st trimester is associated with a lowered risk of developing GDM in later pregnancy. In our unadjusted analysis, this association is not quite statistically significant, however once we adjust for BMI, ethnicity and family history we see the biomarker emerge as a more powerful independent predictor of GDM status (OR of 0.57 for GDM, $\mathrm{p}=0.016$ ). Table 5 demonstrates a significantly lower mean 1,5 AG in those that went on to develop GDM. Fig. 4 is a receiver operating characteristic curve for 1,5 AG levels in the $1 \mathrm{st}$ trimester in a high-risk cohort as a predictor of a screen positive OGTT. Disappointingly here we see no distinct threshold at which 1st trimester 1,5 AG functions with acceptable sensitivity or specificity as a predictor of GDM status (Table 4).

Sex hormone-binding globulin (SHBG) or sex steroid-binding globulin (SSBG) is a glycoprotein that binds to the sex hormones, androgen and estrogen. Levels of SHBG have been shown to decrease with high levels of insulin. Smirnakis at al described the use of SHBG measurement in the first trimester as a predictor of later onset of gestational diabetes [14]. The study evaluated SHBG taken in the first trimester or early in the second trimester in 35

Table 4

OR of a screen positive OGTT at selected thresholds.

\begin{tabular}{|c|c|c|c|}
\hline Biomarker & Odds-Ratio of Screen positive OGTT & $95 \% \mathrm{CI}$ & P-value \\
\hline Adiponectin $\leq 8.9 \mu \mathrm{g} / \mathrm{ml}$ (mean value in our cohort) & 3.3 & $1.6528-6.7$ & $\mathrm{p}=0.0008$ \\
\hline Adiponectin $\leq 12 \mu \mathrm{g} / \mathrm{ml}$ (mean value in healthy general pregnant population) [38] & 1.2 & $0.3297-4.4402$ & $\mathrm{p}=0.77$ \\
\hline
\end{tabular}


Table 5

Mean 1, 5 AG results in gdm and non-GDM patients compared using $t$-test.

\begin{tabular}{llll}
\hline & Mean $\mu \mathrm{g} / \mathrm{mL}$ & SD & p-value \\
\hline Screen Neg (No GDM) & 16.39 & 6.14 & $\mathrm{p}=0.041$ \\
Screen Pos (GDM) & 14.23 & 6.9 & \\
\hline
\end{tabular}

women with subsequent GDM versus 73 controls and 37 patients with an abnormal glucose challenge test. They note that among three different biomarkers examined in the first trimester, SHBG gave the best performance as a predictor of subsequent diagnosis of gestational diabetes. The authors also highlight how SHBG is a test which most hospital laboratories can do readily and at little cost. Maged at al [15] analysed 269 patients of which 27 developed GDM. They found that SHBG at a cut-off value of $211.5 \mathrm{nmol} / \mathrm{L}$ showed a sensitivity and a specificity of $85 \%$ and $37 \%$, respectively. They also looked at high sensitivity C-reactive protein (CRP) and note that when the two biomarkers are combined GDM is predicted with a sensitivity and specificity of $74.07 \%$ and $75.62 \%$, respectively with an overall accuracy of $75.46 \%$. In our study we found that SHBG levels measured in the first trimester were indeed linked to the risk of GDM. However, this was no longer statistically significant once BMI, ethnicity and family history were taken into consideration. It appears that SHBG may not be an independent predictor of GDM status.

Adiponectin is a protein which modulates glucose metabolism by affecting insulin sensitivity. It is secreted both from adipose tissue and the placenta in pregnancy [24]. Adiponectin is antiinflammatory and insulin sensitising. This is achieved by initiating glucose uptake in the skeletal muscle mass and by decreasing glucose production in the liver. Adiponectin levels inversely correlate with body fat percentage and so low levels are seen in obesity which is surprising given that it is produced in adipose tissue. Levels are seen to decline through pregnancy [25] and correlate negatively to BMI and adiposity [39,40]. Low levels of adiponectin have been repeatedly shown to be an independent risk factor for the development of type 2 diabetes [26-28] Low levels of adiponectin are strongly associated with the onset of GDM independently of maternal pre-pregnancy body mass index (BMI) and insulin sensitivity [29-33]. Lain et al. demonstrated in a nested case control study of 59 women (30 of whom developed gestational diabetes) that women with a first trimester adiponectin concentration which was below the 25th percentile were ten times more likely to be diagnosed with GDM when compared to women with higher adiponectin levels [34]. In 2014 $\mathrm{Xu}$ et al. published a systematic review and meta-analysis of 15 studies showing lowered adiponectin levels in GDM patients compared to controls even after adjusting for BMI [35]. To date the available information on adiponectin as a biomarker for GDM has come from small, retrospective case-control or cross-sectional studies. Different sampling sources (maternal, cord or placental) and different assay methods reported have added further complexity. Adiponectin is easily accessible in serum and routine phlebotomy occurs as part of standard antenatal care. Adiponectin has the added benefit of being reproducible despite the fasting or non-fasting state of the subject (up to $72 \mathrm{~h}$ ) [36].

In our study we found that Adiponectin levels in the first trimester are independently linked the risk of GDM later in pregnancy. Higher first-trimester adiponectin levels are associated with a lower risk of subsequent development of GDM. This association appears to be a continuum. There is no clearly identifiable threshold below which GDM is predicated with good sensitivity and specificity.

The strengths of our study include that it is somewhat unique in looking at GDM in a high-risk cohort. We recruited larger numbers than many of the other studies cited above and the data was prospectively collected. One of the inherent limitations of any study of GDM is complicit in the diagnosis. Glucose tolerance is a spectrum and some women who skim below the diagnostic thresholds may still suffer the clinical consequences of uncontrolled hyperglycaemia. The concept of the "fetal glucose steal" phenomenon [37] could potentially affect the results of our study. It is long and well recognised that some patients clinically exhibit the signs of GDM - macrosomic fetus, polyhydramnios and

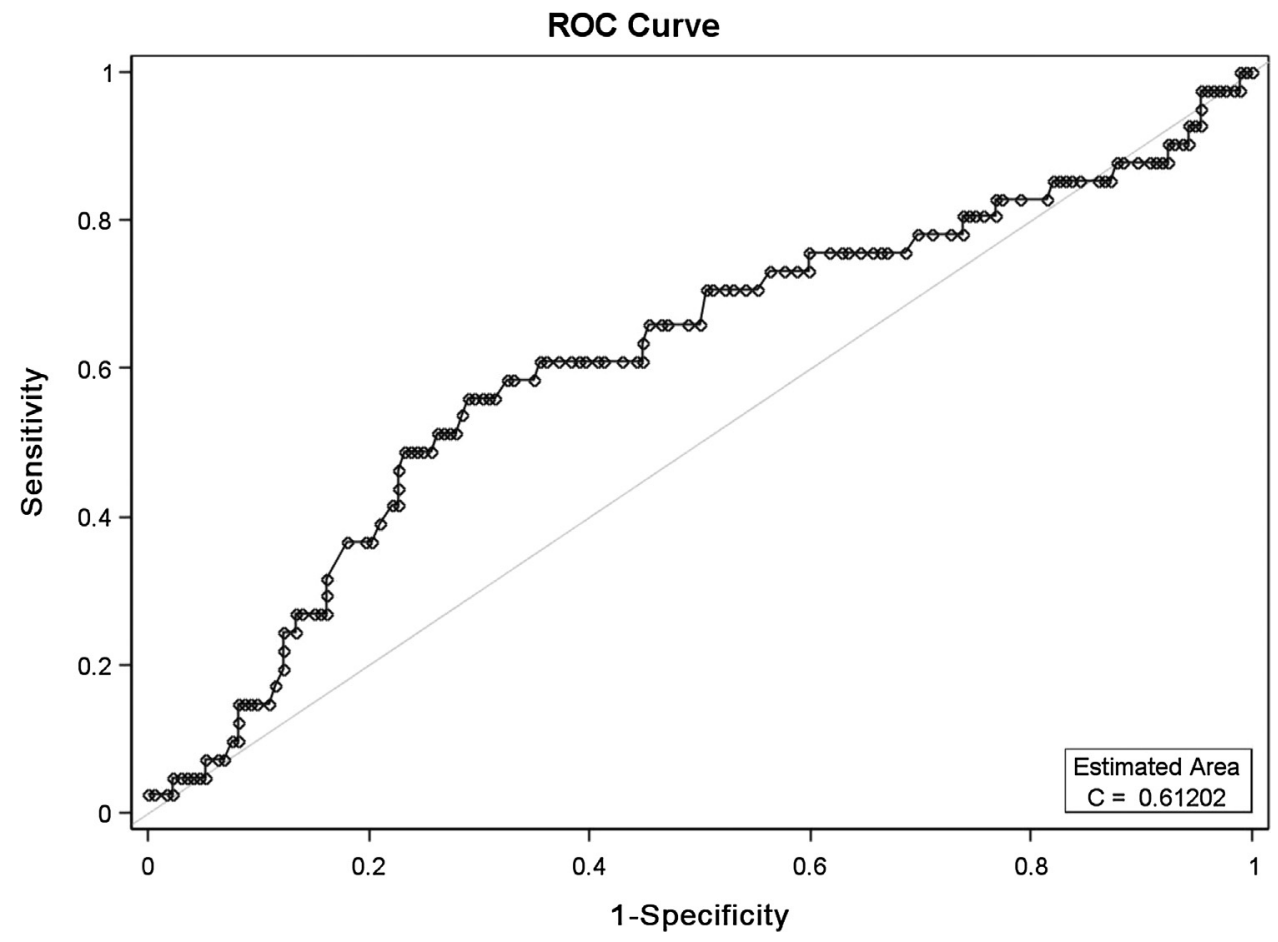

Fig 4. ROC Curve of 1 st trimester 1,5 AG as a predictor of a positive OGTT. 
occasional glycosuria but yet they test negative for GDM when the OGTT is administered. It is postulated that early in gestation when glucose levels have been very high due to maternal insulin deficiency and or resistance, the fetus becomes hyperinsulinaemic. Then at the time of the OGTT, in the late second or early third trimester, since glucose readily and freely crosses the placenta, the fetus metabolises a proportion of it so that the serum glucose levels of the mother are somewhat attenuated and the OGTT is negative. In these cases, therefore, the clinical complications of uncontrolled hyperglycaemia develop in the pregnancy despite a "normal" serum fasting, 1-h and 2-h postprandial glucose level.

In summary Adiponectin levels in the first trimester are independently linked to the risk of GDM. Serum adiponectin $<8.9 \mu \mathrm{g} / \mathrm{ml}$ gives an odds ratio of 3.3 for GDM. After correcting for maternal BMI, ethnicity and family history of diabetes 1,5 AG levels in the first trimester are linked to the likelihood of onset of GDM.

Further work should explore whether women with low adiponectin levels in early pregnancy may benefit from early institution of a diet and lifestyle programme to mitigate against the detrimental effects of GDM.

\section{Acknowledgments}

The author wishes to acknowledge the help of Dr Elizabeth Tully at the Rotunda hospital and also Prof Steve Meany and Philip Stafford of Dublin Institute of Technology. This project was supported by The Friends of the Rotunda Charity.

\section{References}

[1] Crowther CA, Hiller JE, Moss JR, McPhee AJ, Jeffries WS, Robinson JS. Effect of treatment of gestational diabetes mellitus on pregnancy outcomes. N Engl J Med 2005;352:2477-86.

[2] O'Sullivan EP, Avalos G, O'Reilly M, Dennedy MC, Gaffney G, Dunne FP, et al. The prevalence and consequences of gestational diabetes in Ireland. Ir Med J 2012;105(May(5) Suppl):13-5.

[3] Gillespie P, Cullinan J, O'Neill C, Dunne F, ATLANTIC DIP Collaborators. Modelling the independent effects of gestational diabetes mellitus on maternity care and costs. Diabetes Care 2013;36(May(5)):1111-6.

[4] Khalifeh A, Breathnach F, Coulter-Smith S, Robson M, Fitzpatrick C, Malone F. Changing trends in diabetes mellitus in pregnancy. J Obstet Gynaecol 2014;34 (February (2)):135-7.

[5] Langer O, Yogev Y, Most O, Xenakis EM. Gestational diabetes: the consequences of not treating. Am J Obstet Gynecol 2005;192(April(4)):989-97.

[6] Hillier TA, Pedula KL, Vesco KK, Oshiro CE, Ogasawara KK. Impact of maternal glucose and gestational weight gain on child obesity over the first decade of life in normal birth weight infants. Matern Child Health J 2016(May(6)).

[7] Zhang Xun, Decker Adriana, Platt Robert W, Kramer Michael S. How big is too big? The perinatal consequences of fetal macrosomia. Am J Obstet Gynaecol 2008;198(5)e1-e517 Pages 517. e6.

[8] Crowther CA, Hiller JE, Moss JR, McPhee AJ, Jeffries WS, Robinson JS. Effect of treatment of gestational diabetes mellitus on pregnancy outcomes. $\mathrm{N}$ Engl J Med 2005;352:2477-86.

[9] Landon MB, Spong CY, Thom E, Carpenter MW, Ramin SM, Casey B, et al. A multicenter, randomized trial of treatment for mild gestational diabetes. $\mathrm{N}$ Engl J Med 2009;361(October(14)):1339-48.

[10] Moss JR, Crowther CA, Hiller JE, Willson KJ, Robinson JS, Australian Carbohydrate Intolerance Study in Pregnant Women Group. Costs and consequences of treatment for mild gestational diabetes mellitus - evaluation from the ACHOIS randomised trial. BMC Pregnancy Childbirth 2007;28 (October(7)):27.

[12] National Collaborating Centre for Women's and Children's Health. Diabetes in pregnancy: management of diabetes and its complications from preconception to the postnatal period. London, U.K: RCOG Press; 2008.

[13] Nanda S, Savvidou M, Syngelaki A, Akolekar R, Nicolaides KH. Prediction of gestational diabetes mellitus by maternal factors and biomarkers at 11 to 13 weeks. Prenat Diagn 2011;31:135-41.

[14] Smirnakis KV, Plati A, Wolf M, Thadhani R, Ecker JL. Predicting gestational diabetes: choosing the optimal early serum marker. Am J Obstet Gynecol 2007; 196(April(4)):410.

[15] Maged AM, Moety GA, Mostafa WA, Hamed DA. Comparative study between different biomarkers for early prediction of gestational diabetes mellitus. J Matern Fetal Neonatal Med 2014;27(July(11)):1108-12.
[16] Christian LM, Porter K. Longitudinal changes in serum proinflammatory markers across pregnancy and postpartum: effects of maternal body mass index. Cytokine 2014;70(December(2)):134-40.

[17] Ozgu-Erdinc AS, Yilmaz S, Yeral MI, Seckin KD, Erkaya S, Danisman AN. Prediction of gestational diabetes mellitus in the first trimester: comparison of C-reactive protein, fasting plasma glucose, insulin and insulin sensitivity indices. J Maternal-Fetal Neonatal Med 2015;28:1957-62.

[18] Wolf M1, Sandler L, Hsu K, Vossen-Smirnakis K, Ecker JL, Thadhani R. Firsttrimester C-reactive protein and subsequent gestational diabetes. Diabetes Care 2003;26(March(3)):819-24.

[19] Dungan KM1. 5-anhydroglucitol (GlycoMark) as a marker of short-term glycemic control and glycemic excursions. Expert Rev Mol Diagn 2008;8 (January(1)):9-19.

[20] Dworacka M, Wender-Ozegowska E, Winiarska H, Borowska M, Zawiejska A Pietryga $\mathrm{M}$, et al. Plasma anhydro-D-glucitol (1,5-AG) as an indicator of hyperglycaemic excursions in pregnant women with diabetes. Diabet Med 2006;23(February(2)):171-5.

[21] Shani Delaneya S, Yates Coleyb R, Brown Zane. 1, 5-Anhydroglucitol: a new predictor of neonatal birth weight in diabetic pregnancies. Eur J Obst Gynecol Reprod Biol 2015;189(June):55-8.

[22] Nowak N, Skupien J, Cyganek K, Matejko B, Malecki MT. 1, 5-Anhydroglucitol as a marker of maternal glycaemic control and predictor of neonatal birth weight in pregnancies complicated by type 1 diabetes mellitus. Diabetologia 2013;56:709-13.

[23] Tetsuo M. Hamada T, Yoshimatsu K, Lshimatsu J, Matsunaga T. Serum levels of 1, 5-anhydroglucitol during the normal and diabetic pregnancy and puerperium. Acta Obstet Gynecol Scand 1990;69:479-85.

[24] Chen J, Tan B, Karteris E, Zervou S, Digby J, Hillhouse EW, et al. Secretion of adiponectin by human placenta: differential modulation of adiponectin and its receptors by cytokines. Diabetologia 2006;49(June(6)):1292-302.

[25] Catalano PM, Hoegh M, Minium J, Huston-Presley L, Bernard S, Kalhan S, et al. Adiponectin in human pregnancy: implications for regulation of glucose and lipid metabolism. Diabetologia 2006;49(July(7)):1677-85.

[26] Hara K, Horikoshi M, Yamauchi T, Yago H, Miyazaki O, Ebinuma H, et al. Measurement of the high-molecular weight form of adiponectin in plasma is useful for the prediction of insulin resistance and metabolic syndrome. Diabetes Care 2006;29(June(6)):1357-62.

[27] Lara-Castro C, Fu Y, Chung BH, Garvey WT. Adiponectin and the metabolic syndrome: mechanisms mediating risk for metabolic and cardiovascular disease. Curr Opin Lipidol 2007;18(June(3)):263-70.

[28] Vasseur F, Meyre D, Froguel P. Adiponectin, type 2 diabetes and the metabolic syndrome: lessons from human genetic studies. Expert Rev Mol Med 2006;8 (November(27)):1-12.

[29] Altinova AE, Toruner F, Bozkurt N, Bukan N, Karakoc A, Yetkin I, et al. Circulating concentrations of adiponectin and tumor necrosis factor-alpha in gestational diabetes mellitus. Gynecol Endocrinol 2007;23(March(3)):161-5.

[30] Cortelazzi D, Corbetta S, Ronzoni S, Pelle F, Marconi A, Cozzi V, et al. Maternal and foetal resistin and adiponectin concentrations in normal and complicated pregnancies. Clin Endocrinol (Oxf) 2007;66(March(3)):447-53.

[31] Kinalski M1, Telejko B, Kuźmicki M, Kretowski A, Kinalska I. Tumor necrosis factor alpha system and plasma adiponectin concentration in women with gestational diabetes. Horm Metab Res 2005;37(July(7)):450-4.

[32] Ranheim T, Haugen F, Staff AC, Braekke K, Harsem NK, Drevon CA. Adiponectin is reduced in gestational diabetes mellitus in normal weight women. Acta Obstet Gynecol Scand 2004;83(April(4)):341-7.

[33] Retnakaran A, Retnakaran R. Adiponectin in pregnancy: implications for health and disease. Curr Med Chem 2012;19(32):5444-50.

[34] Lain KY1, Daftary AR, Ness RB, Roberts JM. First trimester adipocytokine concentrations and risk of developing gestational diabetes later in pregnancy. Clin Endocrinol (Oxf) 2008;69(September(3)):407-11.

[35] Xu J, Zhao YH, Chen YP, Yuan XL, Wang J, Zhu H, et al. Maternal circulating concentrations of tumor necrosis factor- $\alpha$, leptin, and adiponectin in gestational diabetes mellitus: a systematic review and meta-analysis. Sci World J 2014;2014:926932.

[36] Merl V, Peters A, Oltmanns KM, Kern W, Born J, Fehm HL, et al. Serum adiponectin concentrations during a 72-hour fast in over- and normal-weight humans. Int J Obes 2005;29:998-1001.

[37] Desoye Gernot, Nolan Christopher J. The fetal glucose steal: an underappreciated phenomenon in diabetic pregnancy. Diabetologia 2016;59(June (6)):1089-94

[38] Erikson, et al. Body fat, insulin resistance, energy expenditure and serum concentrations of leptin, adiponectin and resistin before, during and after pregnancy in healthy Swedish women. Br J Nutr 2010;103(January(1)):50-7.

[39] Hauguel-de Mouzon Sylvie, Catalano Patrick. Adiponectin are measurements clinically useful in pregnancy? Diabetes Care 2013;36(June(6)):1434-6.

[40] Lacroix M, Battista M-C, Doyon M, et al. Lower adiponectin levels at first trimester of pregnancy are associated with increased insulin resistance and higher risk of developing gestational diabetes mellitus. Diabetes Care 2013;36:1577-83. 